Revista de Metalurgia

Enero-Marzo 2019, 55 (1) e139

ISSN-L: 0034-8570

https://doi.org/10.3989/revmetalm.139

\title{
Efecto del cobalto sobre nano-partículas de óxido de zinc en el desempeño anticorrosivo de un imprimante alquídico
}

\author{
Santiago Flores ${ }^{\mathrm{a}, \bowtie}$, Oscar Chumpitaz ${ }^{\mathrm{a}}$ \\ ${ }^{a}$ Instituto de Corrosión y Protección, Pontificia Universidad Católica del Perú, Av. Universitaria 1801, San Miguel, Lima 32, Perú \\ ( $\bowtie$ Autor para la correspondencia: sflores@pucp.edu.pe)
}

Enviado: 4 Julio 2018; Aceptado: 13 Febrero 2019; Publicado on-Line: 03 Abril 2019

\begin{abstract}
RESUMEN: El objetivo de esta investigación ha sido estudiar el efecto inhibidor del $\mathrm{CoO}$ en $\mathrm{ZnO}$ obtenidos por síntesis por combustión de solución (SCS) cuando son incorporados a formulaciones alquídicas. ZnO y óxidos mixtos del tipo $\mathrm{Zn}_{1-\mathrm{x}} \mathrm{Co}_{\mathrm{x}} \mathrm{O}$ (donde $\mathrm{x}=0,05-0,10-0,15-0,20$ ) fueron obtenidos por SCS y caracterizados por XRD, ICP-OES, SEM y TEM. Los pigmentos fueron incorporados a imprimantes alquídicos medios en aceite en tres proporciones de $5 \%, 10 \%$ y $20 \%$ en peso pigmentos/resina. Probetas de acero protegidas con las formulaciones alquídicas fueron ensayadas en cámaras de niebla salina y dióxido de azufre. Asimismo, la eficiencia inhibidora de los pigmentos fue estimada mediante ensayos electroquímicos (método de Tafel) en solución $0,1 \mathrm{M}$ de $\mathrm{NaCl}$. Los resultados mostraron que el $\mathrm{CoO}$ mejora las propiedades inhibidoras de los nano-pigmentos base $\mathrm{ZnO}$ obtenidos por SCS.
\end{abstract}

PALABRAS CLAVE: Corrosión; Nanopartículas; Óxido de cobalto; Óxido de zinc; Protección anticorrosiva; Síntesis por combustión de solución

Citar como/Citation: Flores, S.; Chumpitaz, O. (2019). "Efecto del cobalto sobre nano-partículas de óxido de zinc en el desempeño anticorrosivo de un imprimante alquídico". Rev. Metal. 55(1): e139. https://doi.org/10.3989/revmetalm.139.

ABSTRACT: Effect of cobalt on zinc oxide nanoparticles in the anticorrosive performance of an alkyd primer. The objective of this research was to study the inhibitory effect of $\mathrm{CoO}$ in $\mathrm{ZnO}$ obtained by solution combustion synthesis (SCS) when they are incorporated into alkyd formulations. $\mathrm{ZnO}$ and mixed oxides of the type $\mathrm{Zn}_{1-\mathrm{x}} \mathrm{Co}_{\mathrm{x}} \mathrm{O}$ (where $\mathrm{x}=0.05-0.10-0.15-0.20$ ) were obtained by SCS and characterized by XRD, ICP-OES, SEM and TEM. The pigments were incorporated into alkyd primers in three proportions, $5 \%, 10 \%$ and $20 \%$ by weight pigments / resin. Steel probes protected with the alkyd formulations were tested in salt spray and sulfur dioxide chambers. Likewise, the inhibiting efficiency of the pigments was estimated by electrochemical tests (Tafel method) in $0.1 \mathrm{M} \mathrm{NaCl}$ solution. The results showed that $\mathrm{CoO}$ improves the inhibitory properties of the $\mathrm{ZnO}$ based nano-pigments obtained by SCS.

KEYWORDS: Cobalt oxide; Corrosion; Corrosion protection; Nanoparticles; Solution combustion synthesis, Zinc oxide

ORCID: Santiago Flores (https://orcid.org/0000-0001-5683-7182); Oscar Chumpitaz (https://orcid.org/0000-00033268-2854)

Copyright: (C) 2019 CSIC. Este es un artículo de acceso abierto distribuido bajo los términos de la licencia de uso y distribución Creative Commons Reconocimiento 4.0 Internacional (CC BY 4.0). 


\section{INTRODUCCIÓN}

La protección anticorrosiva mediante recubrimientos orgánicos (pinturas anticorrosivas) actúa por diferentes mecanismos: efecto de sacrificio (pinturas ricas en $\mathrm{Zn}$ ), efecto barrera y efecto inhibidor.

$\mathrm{El}$ óxido de Zinc $(\mathrm{ZnO})$ es un pigmento blanco convencional al cual se le atribuye diferentes propiedades, tales como su capacidad de absorción de radiación UV (protegiendo la resina) y sus mecanismos de inhibición catódica y pasivante (Koleske, 1995). Asimismo, es utilizado junto con pigmentos inhibidores activos convencionales como cromato de zinc o borosilicato de calcio aumentando la densidad de entrecruzamiento y dureza de películas de pintura (Koleske, 1995).

Por otro lado, los pigmentos de tamaño convencional para pinturas pueden generar problemas tales como pobre adhesión, flexibilidad reducida, reducción de la resistencia al impacto, a la abrasión o al rayado, y deslaminación prematura. Para superar tales inconvenientes y mejorar su comportamiento el uso de nanopigmentos se ha convertido en una práctica reciente. Debido a su tamaño inherentemente pequeño y a su morfología de partícula muchos de los problemas arriba mencionados pueden ser superados (Cayton y Sawitowski, 2005).

Las nanopartículas más comúnmente usadas en recubrimientos de protección son el $\mathrm{SiO}_{2}, \mathrm{TiO}_{2}$, $\mathrm{ZnO}, \mathrm{Al}_{2} \mathrm{O}_{3}, \mathrm{Fe}_{2} \mathrm{O}_{3}, \mathrm{CaCO}_{3}$, etc.

De acuerdo con Dhoke et al. (2009) se incorporó, a diferentes niveles de carga, nanopartículas de $\mathrm{ZnO}$ en pinturas alquídicas base agua. Los investigadores evaluaron el comportamiento anticorrosivo de los sistemas alquídicos aplicados sobre acero en ensayos de corrosión acelerada en cámara de humedad, niebla salina y exposición a la radiación UV. Asimismo, evaluaron el efecto de las nanopartículas en las propiedades físicas de la película de pintura (dureza, flexibilidad, adhesión e impacto). Dhoke et al. (2009) encontraron que una extremadamente pequeña concentración de nanopartículas de $\mathrm{ZnO}$ puede mejorar la resistencia a la corrosión, al rayado y a la abrasión de la película de pintura. Cabe señalar que en este estudio se utilizaron nanopartículas de $\mathrm{ZnO}(<50 \mathrm{~nm})$ obtenidas comercialmente. Un estudio paralelo del mismo grupo de investigación con nanopartículas de $\mathrm{Fe}_{2} \mathrm{O}_{3}$ reportó resultados similares (Dhoke y Kanna, 2009a; Dhoke y Kanna, 2009b).

El efecto de nanopartículas de $\mathrm{ZnO}$ sobre la protección anticorrosivas de otros tipos genéricos de pinturas, diferentes a los sistemas alquídicos ha sido estudiado recientemente. Ramenzazadeh et al. (2011) estudiaron el efecto de diferentes cargas $(2 ; 3,5 ; 5$ y $6,5 \%)$ de nanopartículas de $\mathrm{ZnO}$ sobre la protección anticorrosiva brindada por una película de pintura epoxi-poliamida. Probetas de acero protegidas con el sistema epóxico fueron sumergidas en solución $\mathrm{NaCl} 3,5 \%$ en peso, durante 1344 h.
El comportamiento anticorrosivo fue monitoreado aplicando espectroscopía de impedancia electroquímica (IES). Los resultados indicaron que, a pesar de la reducción del entrecruzamiento del sistema epóxico debido a la presencia de las nanopartículas de $\mathrm{ZnO}$, las propiedades anticorrosivas de la película epóxica fue considerablemente mejorada. Esto fue atribuido a dos razones. Primero, el tamaño de las partículas de $\mathrm{ZnO}$ mejora la protección por efecto barrera contra la difusión de los agentes agresivos hacia el sustrato. Segundo, las nanopartículas pueden aumentar la resistencia contra la degradación hidrolítica (Ramenzanzadeh et al., 2011) y la hidrofobicidad (Ammar et al., 2016a). Rashvand y Ranjbar (2013) realizaron un estudio similar en pinturas de poliuretano base agua. Las nanopartículas de $\mathrm{ZnO}$, obtenidas comercialmente e incorporadas al 3\% en peso en el recubrimiento de poliuretano, mejoraron la resistencia a la corrosión en 2 órdenes de magnitud en condiciones de inmersión en $\mathrm{NaCl}$ 3,5\% cuando es evaluado mediante EIS. Finalmente, (Ammar et al., 2016b) estudiaron la incorporación de nanopartículas de $\mathrm{ZnO}$ en un recubrimiento a base de resinas acrílica y de silicona, para lo cual fue comprobado su muy destacado efecto barrera y superior habilidad anticorrosiva.

Sin embargo, el efecto beneficioso del $\mathrm{ZnO}$ puede ser mejorado mediante su modificación con otros óxidos, como el óxido de cobalto. Rasouli y Danaee (2011) prepararon un óxido mixto de $\mathrm{Zn}$ y $\mathrm{Co}, \mathrm{Zn}_{0.9} \mathrm{Co}_{0,10}$, mediante un procedimiento de síntesis cerámico y otro método de síntesis por combustión en solución (SCS). Los pigmentos, caracterizados por XRD y FTIR, fueron incorporados a un recubrimiento alquídico en un porcentaje del 2\% empleando agitación mecánica seguido de ultrasonificación. Los sistemas alquídicos fueron aplicados sobre sustratos de acero, y evaluados en condiciones de inmersión en $\mathrm{NaCl} 3,5 \%$ mediante técnicas de polarización electroquímica e impedancia electroquímica. Concluyeron que la combinación de los óxidos de cobalto y zinc mejoran las propiedades anticorrosivas del pigmento obtenido. Los investigadores encontraron que el método de preparación de los pigmentos de $\mathrm{ZnO}-\mathrm{CoO}$ pueden influenciar las características de las partículas obtenidas tales como el tamaño de partícula y la forma. Así, el nanopigmento preparado mediante síntesis por combustión en solución (SCS) logro una mejor resistencia a la corrosión respecto al pigmento sintetizado mediante reacciones de estado sólido (métodos cerámicos).

Los óxidos de color verde obtenidos fueron considerados interesantes por ser más respetuosos del medio ambiente que los pigmentos a base de cromo (Rasouli y Danaee, 2011). La USEPA (United States Environmental Protection Agency) ha elaborado una lista de contaminantes del aire (USEPA, 2018), entre ellos el cobalto (USEPA, 2000a) 
y cromo (USEPA, 2000b), donde es clara la menor peligrosidad del cobalto según datos de exposición a la inhalación.

Ahmed y Selim (2005) realizaron un estudio en sistemas alquídicos probando varias relaciones de óxidos en el pigmento $\mathrm{ZnO}-\mathrm{CoO}(5,10,15$ y $20 \%$ de $\mathrm{CoO}$ ) elaborados por procedimientos cerámicos. Los ensayos de corrosión acelerada e impedancia electroquímica revelaron que la presencia de cobalto mejora la acción de óxido de zinc en una mutua interacción que conduce a una mejor inhibición de la corrosión del sustrato de acero.

Resumiendo, según los estudios realizados, por un lado, se tiene que existe un creciente interés por evaluar el efecto de las nanopartículas de $\mathrm{ZnO}$ sobre las propiedades anticorrosivas de diferentes tipos de pinturas (alquídicas, epóxicas, poliuretano). Por otro lado, se ha encontrado un efecto beneficioso del cobalto $(\mathrm{CoO})$ al modificar el $\mathrm{ZnO}$ cuando es elaborado el óxido mixto nanométrico mediante SCS. La SCS es un proceso versátil, simple y rápido que permite la elaboración efectiva de una variedad de nanomateriales uni o multicomponentes con morfología prediseñada (Patil, 1993; Patil et al., 1997; Abu Ayana et al., 1997; Patil et al., 2008; Aruna y Mukasyan, 2008).

Por ello, la propuesta de la presente investigación busca estudiar el efecto anticorrosivo de nanopigmentos mixtos de $\mathrm{ZnO}-\mathrm{CoO}$, obtenidos mediante $\mathrm{SCS}$ en varias relaciones $\mathrm{ZnO} / \mathrm{CoO}$, en imprimantes alquídicos evaluados mediante ensayos de corrosión acelerada.

\section{MATERIALES Y MÉTODOS}

\subsection{Síntesis de los óxidos por combustión en solución}

Los óxidos fueron sintetizados en base a nitrato de zinc $\left(\mathrm{Zn}\left(\mathrm{NO}_{3}\right)_{2} \cdot 6 \mathrm{H}_{2} \mathrm{O}\right)$ y nitrato de cobalto $\left(\mathrm{Co}\left(\mathrm{NO}_{3}\right)_{2} \cdot 6 \mathrm{H}_{2} \mathrm{O}\right)$ como oxidantes, y Glicina $\left(\mathrm{NH}_{2} \mathrm{CH}_{2} \mathrm{CO}_{2} \mathrm{H}\right)$ como combustible. Todos fueron reactivos Sigma-Aldrich.

Las sales fueron pesadas en un vaso de precipitados. Posteriormente, fueron mezcladas con calor y agua hasta que la solución formada muestre una consistencia viscosa.

La mezcla fue llevada a una mufla precalentada a $500{ }^{\circ} \mathrm{C}$ para que, después de $2 \mathrm{~min}$ aproximadamente, se lleve a cabo la combustión. En dicho instante la temperatura en la vecindad de la reacción supera los $1000{ }^{\circ} \mathrm{C}$; debido a la forma porosa, de mayor volumen aparente que toma el producto de reacción, son formadas nanopartículas muy puras de óxidos.

Los óxidos mixtos sintetizados por combustión en solución tuvieron la siguiente estequiometría: $\mathrm{Zn}_{0,95} \mathrm{Co}_{0.05} \mathrm{O}, \mathrm{Zn}_{0,90} \mathrm{Co}_{0,10} \mathrm{O}, \mathrm{Zn}_{0,85} \mathrm{Co}_{0.15} \mathrm{O}$ y $\mathrm{Zn}_{0,80} \mathrm{Co}_{0,20} \mathrm{O}$. Asimismo, fue elaborado $\mathrm{ZnO}$ puro por SCS.

\subsection{Análisis de los óxidos mixtos por Difracción de Rayos X}

Cinco muestras de óxidos (1-1,5 g) fueron analizadas: $\mathrm{ZnO}$ producido por SCS, óxidos mixtos producidos por SCS con la siguiente estequiometría: $\mathrm{Zn}_{0,95} \mathrm{Co}_{0,05} \mathrm{O}, \mathrm{Zn}_{0,90} \mathrm{Co}_{0,10} \mathrm{O}, \mathrm{Zn}_{0,85} \mathrm{Co}_{0,15} \mathrm{O}$ y $\mathrm{Zn}_{0,80} \mathrm{Co}_{0,20} \mathrm{O}$.

El análisis fue realizado en un Difractómetro marca BRUKER, modelo D8-FOCUS. Se empleó un tubo de $\mathrm{Cu}$ cuya longitud de onda, correspondiente a $\mathrm{Ka} 1-\mathrm{Cu}$, es $1=1.5406 \mathrm{~A}$. Las condiciones de medición fueron las siguientes: Rango angu-

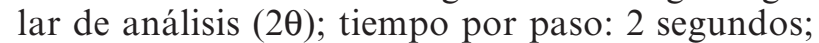
voltaje de salida del tubo $=40 \mathrm{kV}$; corriente de salida del tubo $=40 \mathrm{~mA}$; detector con contador de centelleo.

\subsection{Análisis químico de los óxidos}

Fueron cuantificados el zinc y cobalto mediante ICP-OES, inductively coupled plasma optical emission spectrometry, según método basado en "Método EPA 200.7, 1994, Rev. 4.4". Para la realización de los ensayos las soluciones formadas a partir de las muestras fueron diluidas doscientas veces.

\subsection{Análisis de los óxidos por Microscopía Electrónica de Transmisión (TEM)}

Las muestras fueron estudiadas mediante TEM utilizando un microscopio marca Philips, modelo CM20-Ultra Twin, el cual es operado a $200 \mathrm{kV}$. La preparación de muestra consistió en colocar gotas de solución de nanopartículas en un portamuestra de oro que estaba recubierto de carbono.

\subsection{Formulación de imprimantes alquídicos}

El recubrimiento alquídico utilizado estuvo basado en una formulación convencional en la cual el talco fue reemplazado, de manera parcial, por los pigmentos estudiados (Tabla 1).

Todas las formulaciones estuvieron basadas en una resina alquídica media en aceite. Las pinturas

TABla 1. Pigmentos utilizados en las formulaciones alquídicas

\begin{tabular}{c}
\hline Pigmento \\
\hline Óxido de zinc commercial \\
$\mathrm{ZnO}$ sintetizado por SCS \\
$\mathrm{Zn}_{0,95} \mathrm{Co}_{0,05} \mathrm{O}$ sintetizado por SCS \\
$\mathrm{Zn}_{0,90} \mathrm{Co}_{0,10} \mathrm{O}$ sintetizado por SCS \\
$\mathrm{Zn}_{0,85} \mathrm{Co}_{0,15} \mathrm{O}$ sintetizado por SCS \\
$\mathrm{Zn}_{0,80} \mathrm{Co}_{0,20} \mathrm{O}$ sintetizado por SCS \\
\hline
\end{tabular}


fueron divididas en tres grupos de $5 \%$ (a), $10 \%$ (b) y $20 \%$ (c) en peso de pigmento estudiado respecto del peso de la formulación. Cada grupo estuvo formado por 05 pinturas formuladas con el pigmento $\mathrm{ZnO}$ sintetizado por SCS y los óxidos mixtos $\mathrm{Zn}_{1-\mathrm{x}} \mathrm{Co}_{\mathrm{x}} \mathrm{O}$, donde $\mathrm{x}=0,05 ; 0,10 ; 0,15$ y 0,20 (Tabla 1 ); el $\mathrm{ZnO}$ comercial solo fue utilizado al $10 \%$ en peso. Finalmente, los pigmentos estudiados fueron incorporados a 15 formulaciones y 01 formulación blanco con $\mathrm{ZnO}$ comercial.

Al ser incorporados a la formulación, los pigmentos sintetizados fueron ultrasonificados por $8 \mathrm{~min}$ en agua $\left(100 \mathrm{~g} / 150 \mathrm{~mL} \mathrm{H}_{2} \mathrm{O}\right)$. El equipo utilizado fue el VCX 500 Vibracell de Sonics \& Materials Inc. Posteriormente, el agua fue eliminada por evaporación lenta.

Todas las pinturas, elaboradas con dispersora de laboratorio, son presentadas en la Tabla 2, con los contenidos de resina, solvente, cargas, pigmentos y aditivos. La viscosidad de las pinturas alquídicas (Tabla 3) fue determinada en base al método descrito en la norma ASTM D 562-10 (2014) utilizando el Viscosímetro KU-2 de BYK.

\subsection{Ensayos de corrosión acelerada}

Probetas de $10 \times 15 \mathrm{~cm}$ de acero estructural fueron desengrasadas y chorreadas hasta metal blanco (Asa3) de acuerdo con la norma ISO 8501-1. Las probetas chorreadas fueron recubiertas empleando pincel con las pinturas alquídicas medias en aceite formuladas con $\mathrm{ZnO}$ y óxidos mixtos $\mathrm{Zn}_{1-\mathrm{x}} \mathrm{Co}_{\mathrm{x}} \mathrm{O}$ elaborados por SCS, además de $\mathrm{ZnO}$ comercial. Los espesores de película seca, según la norma ASTM B 499-09 (2014), de los imprimantes alquídicos son presentados en la Tabla 4.

Las probetas pintadas fueron ensayadas usando: i) exposición en cámara de niebla salina basada en la norma ASTM B 117-16 (2016) por $863 \mathrm{~h}$; y ii) exposición en cámara de dióxido de azufre basada en la norma ISO 3231 por $3528 \mathrm{~h}$. A lo largo de cada ensayo el grado de corrosión fue

TABLA 2. Formulaciones de pinturas con los óxidos mixtos $\mathrm{Zn}_{1-\mathrm{x}} \mathrm{Co}_{\mathrm{x}} \mathrm{O}$, donde $\mathrm{x}=0,05 ; 0,10 ; 0,15$ y 0,20 basados en resina alquídica media en aceite

\begin{tabular}{|c|c|c|c|c|c|c|c|c|c|c|c|c|c|c|c|c|}
\hline \multirow[b]{2}{*}{ Componente (\%) } & \multicolumn{16}{|c|}{ Formulaciones } \\
\hline & 1 & 2 & 3 & 4 & 5 & 6 & 7 & 8 & 9 & 10 & 11 & 12 & 13 & 14 & 15 & 16 \\
\hline Resina de Soya & \multicolumn{16}{|c|}{30,0} \\
\hline Aguarrás & \multicolumn{16}{|c|}{27,2} \\
\hline Bentone & \multicolumn{16}{|c|}{0,3} \\
\hline Dióxido de titanio & \multicolumn{16}{|c|}{11,0} \\
\hline Disperbyk 108 & \multicolumn{16}{|c|}{0,1} \\
\hline Talco & 20,5 & 25,5 & 20,5 & 10,5 & 25,5 & 20,5 & 10,5 & 25,5 & 20,5 & 10,5 & 25,5 & 20,5 & 10,5 & 25,5 & 20,5 & 10,5 \\
\hline Óxido de zinc comercial & 10,0 & -- & -- & -- & -- & -- & -- & -- & -- & -- & -- & -- & -- & -- & -- & -- \\
\hline $\mathrm{ZnO}$ & -- & 5,0 & 10,0 & 20,0 & -- & -- & -- & -- & -- & -- & -- & -- & -- & -- & -- & -- \\
\hline $\mathrm{Zn}_{0,95} \mathrm{Co}_{0,05} \mathrm{O}$ & -- & -- & -- & -- & 5,0 & 10,0 & 20,0 & -- & -- & -- & -- & -- & -- & -- & -- & -- \\
\hline $\mathrm{Zn}_{0,9} \mathrm{Co}_{0,10} \mathrm{O}$ & -- & -- & -- & -- & -- & -- & -- & 5,0 & 10,0 & 20,0 & -- & -- & -- & -- & -- & -- \\
\hline $\mathrm{Zn}_{0,85} \mathrm{Co}_{0,15} \mathrm{O}$ & -- & -- & -- & -- & -- & -- & -- & -- & -- & -- & 5,0 & 10,0 & 20,0 & -- & -- & -- \\
\hline $\mathrm{Zn}_{0,80} \mathrm{Co}_{0,20} \mathrm{O}$ & -- & -- & -- & -- & -- & -- & -- & -- & -- & -- & -- & -- & -- & 5,0 & 10,0 & 20,0 \\
\hline Octoato de plomo & \multicolumn{16}{|c|}{0,4} \\
\hline Secante a base de cobalto & \multicolumn{16}{|c|}{0,1} \\
\hline Secante a base de calcio & \multicolumn{16}{|c|}{0,2} \\
\hline Aditivo Antipiel & \multicolumn{16}{|c|}{0,2} \\
\hline Total & 100 & 100 & 100 & 100 & 100 & 100 & 100 & 100 & 100 & 100 & 100 & 100 & 100 & 100 & 100 & 100 \\
\hline
\end{tabular}

TABLA 3. Viscosidad, según la norma ASTM D 562-10 (2014), de las pinturas alquídicas medias en aceite

\begin{tabular}{lrrrrrrrrrrrrrrrr}
\hline & \multicolumn{11}{c}{ Formulaciones } \\
\hline & $\mathbf{1}$ & $\mathbf{2}$ & $\mathbf{3}$ & $\mathbf{4}$ & $\mathbf{5}$ & $\mathbf{6}$ & $\mathbf{7}$ & $\mathbf{8}$ & $\mathbf{9}$ & $\mathbf{1 0}$ & $\mathbf{1 1}$ & $\mathbf{1 2}$ & $\mathbf{1 3}$ & $\mathbf{1 4}$ & $\mathbf{1 5}$ & $\mathbf{1 6}$ \\
\hline Temperatura en la medición $\left({ }^{\circ} \mathbf{C}\right)$ & 31 & 33 & 32 & 31 & 32 & 31 & 30 & 32 & 32 & 30 & 31 & 32 & 30 & 30 & 30 & 30 \\
Viscosidad (KU) & 87,0 & 80,3 & 79,1 & 81,2 & 83,5 & 84,3 & 76,8 & 87,3 & 80,3 & 82,7 & 85,7 & 79,7 & 86,3 & 88,3 & 80,1 & 83,8 \\
\hline
\end{tabular}


TABLA 4. Espesores de película seca, según la norma ASTM B 499-09 (2014), de los imprimantes alquídicos aplicados sobre probetas chorreadas de acero estructural

\begin{tabular}{llcc}
\hline \% wpig./wpaint & \multicolumn{1}{c}{ Pigmento } & $\begin{array}{c}\text { Espesor } \\
(\boldsymbol{\mu m})\end{array}$ & $\begin{array}{c}\text { Desviación } \\
\text { estándar }(\boldsymbol{\mu m})\end{array}$ \\
\hline 5 & $\mathrm{ZnO}$ & 59,44 & 3,397 \\
& $\mathrm{Zn}_{0,95} \mathrm{Co}_{0,05} \mathrm{O}$ & 60,31 & 3,658 \\
& $\mathrm{Zn}_{0,90} \mathrm{Co}_{0,10} \mathrm{O}$ & 62,77 & 3,044 \\
& $\mathrm{Zn}_{0,85} \mathrm{Co}_{0,15} \mathrm{O}$ & 63,57 & 4,727 \\
& $\mathrm{Zn}_{0,80} \mathrm{Co}_{0,20} \mathrm{O}$ & 69,04 & 4,591 \\
& $\mathrm{ZnO}$ comercial & 57,05 & 3,137 \\
& $\mathrm{ZnO}$ & 57,28 & 4,681 \\
& $\mathrm{Zn}_{0,95} \mathrm{Co}_{0,05} \mathrm{O}$ & 58,98 & 3,809 \\
& $\mathrm{Zn}_{0,90} \mathrm{Co}_{0,10} \mathrm{O}$ & 64,64 & 6,889 \\
& $\mathrm{Zn}_{0,85} \mathrm{Co}_{0,15} \mathrm{O}$ & 63,05 & 3,689 \\
& $\mathrm{Zn}_{0,80} \mathrm{Co}_{0,20} \mathrm{O}$ & 75,32 & 3,142 \\
& $\mathrm{ZnO}$ & 66,75 & 5,308 \\
& $\mathrm{Zn}_{0,95} \mathrm{Co}_{0,05} \mathrm{O}$ & 58,78 & 3,720 \\
& $\mathrm{Zn}_{0,90} \mathrm{Co}_{0,10} \mathrm{O}$ & 57,62 & 3,727 \\
& $\mathrm{Zn}_{0,85} \mathrm{Co}_{0,15} \mathrm{O}$ & 56,15 & 3,114 \\
& $\mathrm{Zn}_{0,80} \mathrm{Co}_{0,20} \mathrm{O}$ & 69,46 & 6,545 \\
\hline
\end{tabular}

evaluado según las normas ASTM D 610-08 (2012) y ASTM D 714-02 (2009).

\subsection{Ensayos Electroquímicos}

Para los ensayos fueron utilizadas probetas de acero dulce JIS G3141 (0,15\% en Carbono, $0,60 \%$ en Manganeso, $0,05 \%$ en Azufre y 0,10\% en Fósforo), SPCC grade $(2 \mathrm{~cm} \times 6 \mathrm{~cm} \times 3 \mathrm{~mm})$, las cuales fueron preparadas superficialmente con el uso de una pulidora, modelo METASERV ${ }^{\mathrm{TM}}$ y con papeles abrasivos $120,140,400$ y 600 y, finalmente, desengrasadas con etanol. Un área de $1 \mathrm{~cm}^{2}$, aproximadamente, fue delimitada con parafina.

Fue utilizada una celda electroquímica de tres electrodos: un electrodo de cloruro de plata $(\mathrm{Ag} / \mathrm{AgCl} / \mathrm{KCl} 3 \mathrm{M})$ como referencia, una probeta de acero dulce (ver párrafo anterior) como el electrodo de trabajo y un contraelectrodo de Platino como placa de $0,8 \times 0,8 \mathrm{~cm}$. Las mediciones fueron realizadas en suspensiones del pigmento $(1 \mathrm{~g}$ de pigmento / $100 \mathrm{~mL}$ de solución). El electrolito utilizado fue una solución de $\mathrm{NaCl}$ 0,1M. La amplitud del barrido fue desde $-250 \mathrm{mV}$ hasta $250 \mathrm{mV}$ versus OCP, a una velocidad de barrido de $1 \mathrm{mVs}^{-1}$.

Fue utilizado el método de Tafel para la determinación de las densidades de corriente (Icorr) de probetas de acero en suspensiones de los pigmentos estudiados. Los ensayos fueron realizados después de $24 \mathrm{~h}$ de inmersión en agitación constante. Posteriormente, fue calculada la eficiencia de inhibición de cada pigmento ( $\eta p$ ) con ensayos adicionales de muestras blanco (sin pigmento) y en base a la ecuación (1):

$$
\eta \mathrm{p}(\%)=\left[\frac{\text { icorr }(\sin \text { pigmento })-\text { Icorr }(\text { spigmento })}{i \operatorname{corr}(\sin \text { pigmento })}\right] \times 100
$$

\section{RESULTADOS}

- Análisis de los óxidos por difracción de rayos X:

Los difractogramas de Rayos $\mathrm{X}$ presentados en las Figs. 1 y 2 para los óxidos $\mathrm{ZnO}$ y $\mathrm{Zn}_{1-\mathrm{x}} \mathrm{Co}_{\mathrm{x}} \mathrm{O}$, muestran que los valores de ángulo de difracción, $2 \theta$, y raíz cuadrada de la intensidad relativa, $\sqrt{ } \mathrm{I} / \mathrm{I}_{0}$, permiten identificar dos fases cristalinas, $\mathrm{ZnO}$ y $\mathrm{Co}_{3} \mathrm{O}_{4}$, en los óxidos mixtos.

- Análisis químico de los óxidos:

Fueron comparados los contenidos teóricos, de zinc y cobalto en los óxidos según la estequiometría, con los resultados obtenidos por ICP-OES en los óxidos sintetizados. En la Tabla 5 es presentada la relación teórica molar $\mathrm{Zn} / \mathrm{Co}$ (p.e. $0,95 / 0,05=19$ ) y la relación real molar $\mathrm{Zn} / \mathrm{Co}$ obtenida a partir del análisis mediante ICP-OES.

- Análisis de los óxidos por microscopía electrónica de transmisión:

El estudio por microscopía electrónica de transmisión permitió establecer, como puede ser apreciado en la Fig. 3, el tamaño y la morfología de las partículas (en este caso del $\mathrm{ZnO}$ ).

- Ensayos de corrosión acelerada:

En la Tabla 6 son presentados el grado de ampollamiento, según la norma ASTM D 714-02 (2009), y el grado de corrosión, según la norma ASTM D 610-08 (2012) de los sistemas evaluados para ensayos en niebla salina neutra ( $863 \mathrm{~h}$ ) y dióxido de azufre $(3528 \mathrm{~h})$.

- Ensayos electroquímicos:

En la Tabla 7 se puede apreciar los valores de Icorr y eficiencia de inhibición para los pigmentos estudiados.

\section{DISCUSIÓN}

- Análisis de los óxidos por difracción de rayos X:

La identificación de dos fases cristalinas, $\mathrm{ZnO}$ y $\mathrm{Co}_{3} \mathrm{O}_{4}$, en los óxidos mixtos no está de acuerdo con lo reportado por Ahmed y Selim (2005) quienes afirmaron que aproximadamente $20 \%$ de $\mathrm{CoO}$ puede ser retenido en la solución sólida de la fase Wurzita. Cabe señalar que el método de preparación utilizado por estos investigadores fue síntesis por calcinación. 
(a)

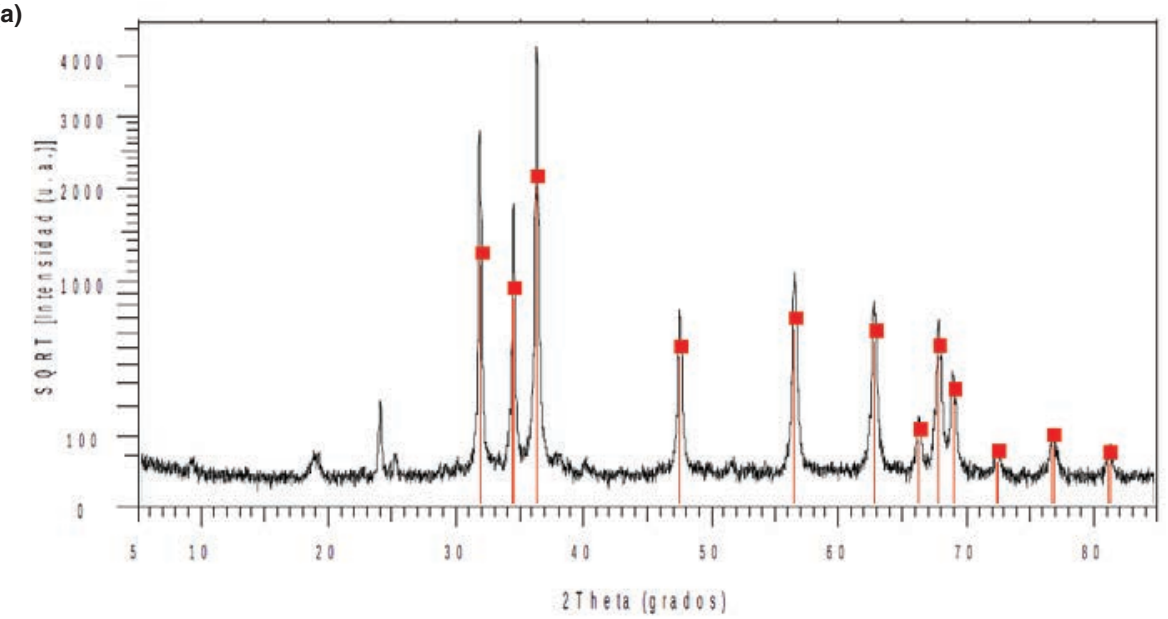

(b)

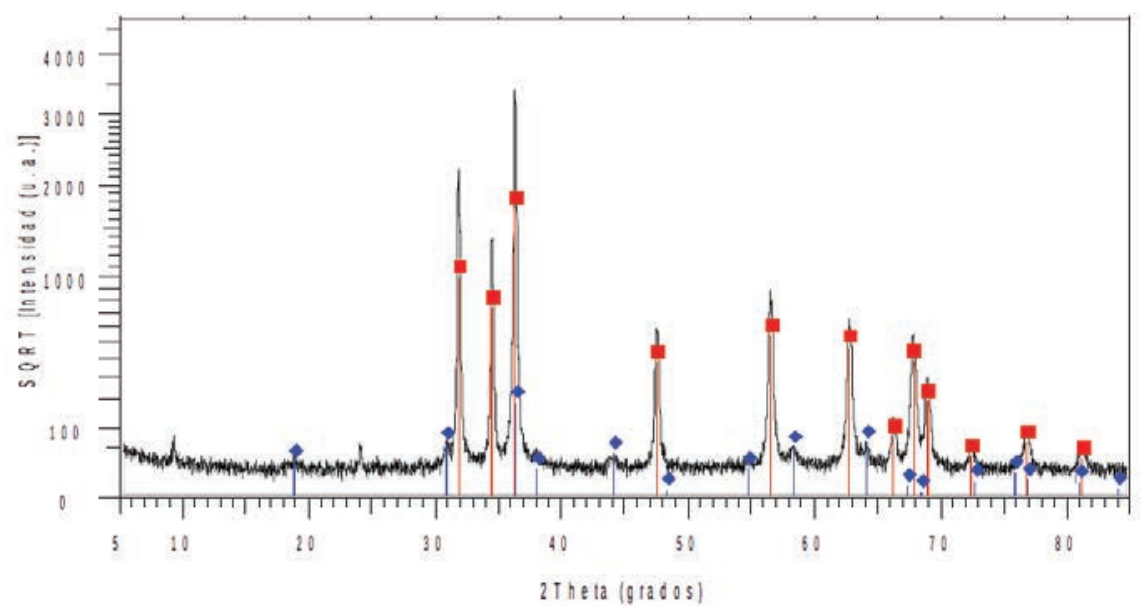

(c)

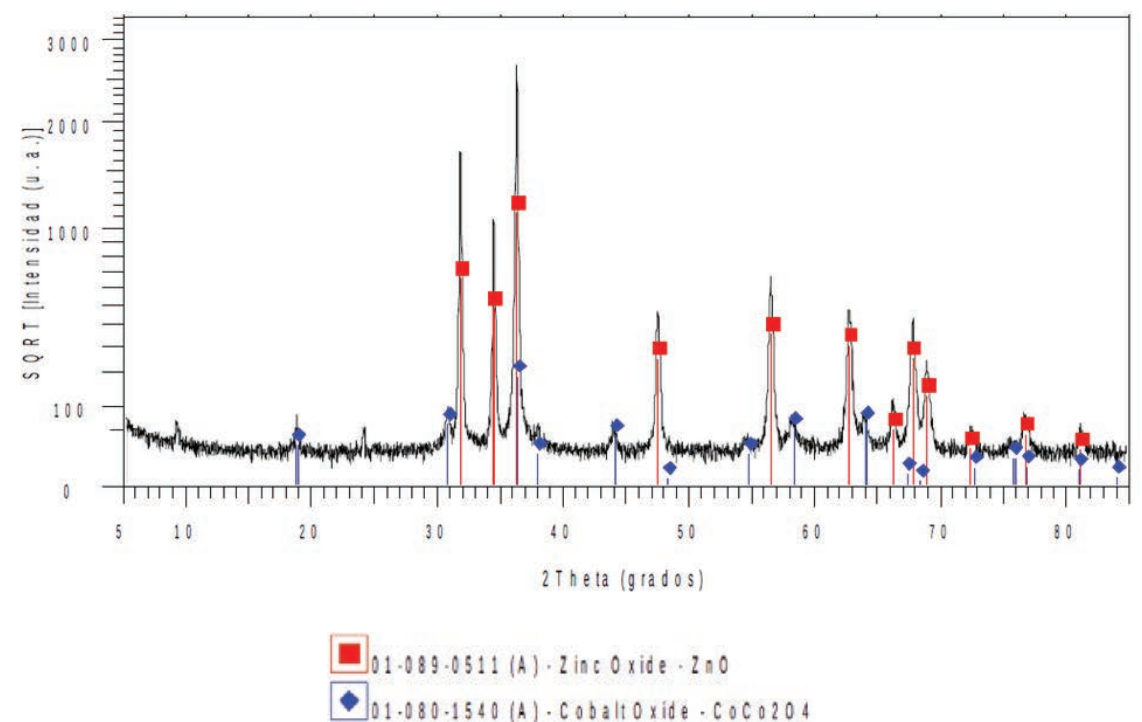

Figure 1. Difractogramas XRD para los pigmentos $\mathrm{ZnO}$ (a), $\mathrm{Zn}_{0,95} \mathrm{Co}_{0,05} \mathrm{O}$ (b) y $\mathrm{Zn}_{0,90} \mathrm{Co}_{0,10} \mathrm{O}$ (c) obtenidos por SCS. 
Efecto del cobalto sobre nano-partículas de óxido de zinc en el desempeño anticorrosivo de un imprimante alquídico 7

(a)

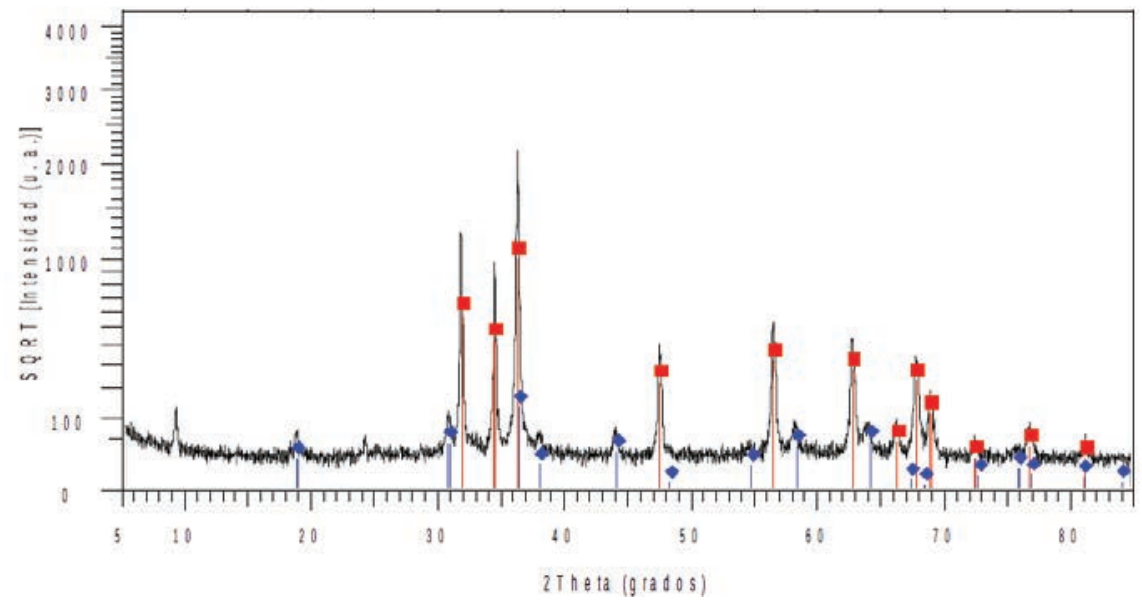

(b)

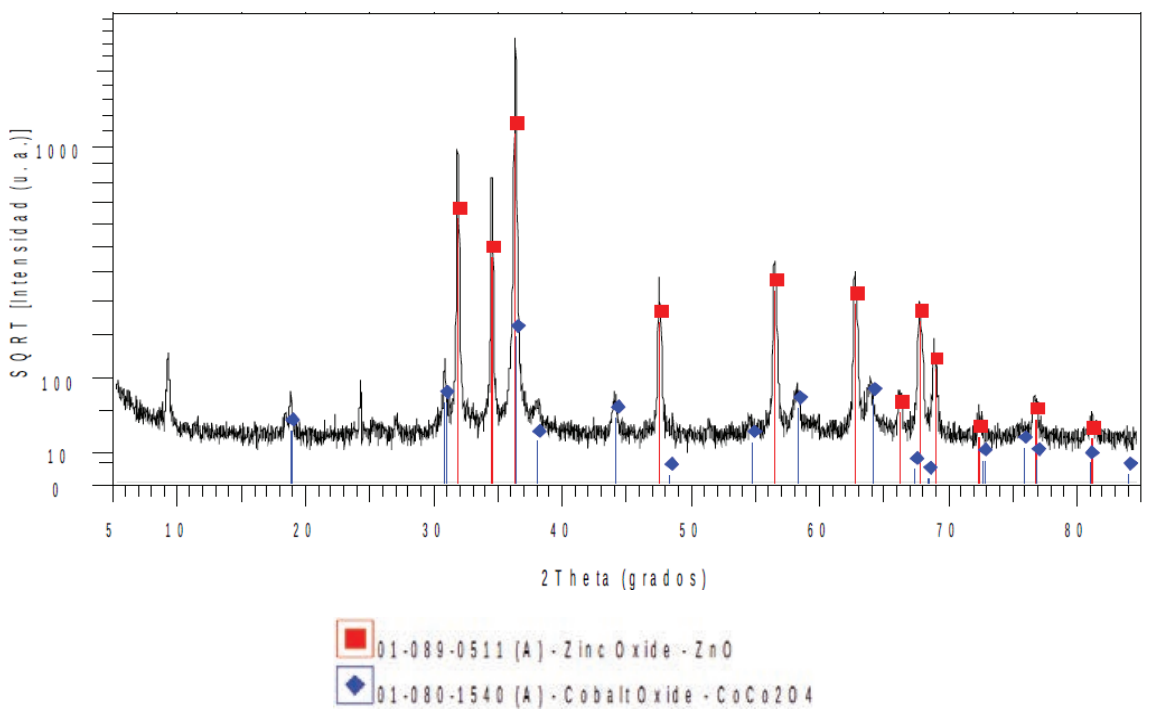

Figure 2. Difractogramas XRD para los pigmentos $\mathrm{Zn}_{0,85} \mathrm{Co}_{0,15} \mathrm{O}$ (a) y $\mathrm{Zn}_{0,80} \mathrm{Co}_{0,20} \mathrm{O}$ (b) obtenidos por $\mathrm{SCS}$.

TABLA 5. Relación teórica molar Zn/Co y relación real molar Zn/Co obtenida a partir del análisis mediante ICP-OES

\begin{tabular}{lcc}
\hline Óxido & Teórica & Real \\
\hline $\mathrm{Zn}_{0,95} \mathrm{Co}_{0,05} \mathrm{O}$ & 19 & 20,07 \\
$\mathrm{Zn}_{0,90} \mathrm{Co}_{0,10} \mathrm{O}$ & 9 & 9,21 \\
$\mathrm{Zn}_{0,85} \mathrm{Co}_{0,15} \mathrm{O}$ & 5,67 & 5,77 \\
$\mathrm{Zn}_{0,80} \mathrm{Co}_{0,20} \mathrm{O}$ & 4 & 4,12 \\
\hline
\end{tabular}

Sin embargo, Ahmed and Selim (2005) encontraron que el óxido $20 \% \mathrm{CoO} . \mathrm{ZnO}$ mostró la presencia de pequeñas líneas en los difractogramas relacionadas con la fase cristalina $\mathrm{CoO}$, como ha sido observado en este estudio para todos los óxidos mixtos sintetizados por SCS.
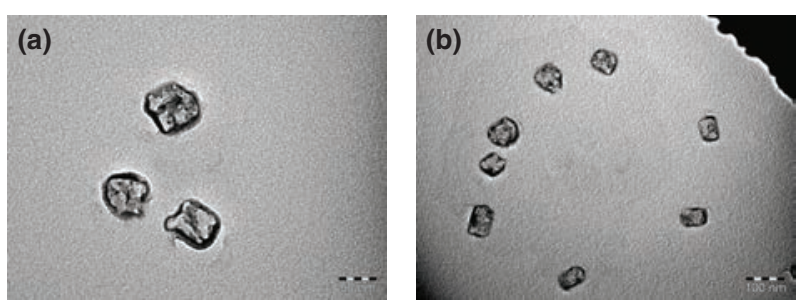

FIgure 3. Morfología de $\mathrm{ZnO}$ obtenido por SCS. a) Morfología irregular, pero cuasi esférica del $\mathrm{ZnO}$; b) partículas de $\mathrm{ZnO}$ donde se observa la homogeneidad en el tamaño de las partículas que están por debajo de los $100 \mathrm{~nm}$.

- Análisis químico de los óxidos:

Los resultados confirmaron con buena aproximación los contenidos esperados de zinc y cobalto en los óxidos preparados. 
TABLA 6. Evaluación de probetas para ensayos en niebla salina neutra y dióxido de azufre

\begin{tabular}{|c|c|c|c|c|c|c|c|}
\hline \multirow{2}{*}{$\begin{array}{l}\text { Formulación } \\
\left(w_{\text {pigmento }} / w_{\text {pintura }} \times\right. \\
100)\end{array}$} & \multirow{2}{*}{$\begin{array}{l}\text { Pigmento } \\
\text { estudiado }\end{array}$} & \multicolumn{3}{|c|}{ Niebla Salina } & \multicolumn{3}{|c|}{ Dióxido de Azufre } \\
\hline & & Ampollamiento & Corrosión & Foto & Ampollamiento & Corrosión & Foto \\
\hline \multirow[t]{5}{*}{5} & $\mathrm{ZnO}$ & $4 \mathrm{M}$ & $5 \mathrm{P}$ & & $4 \mathrm{MD}$ & $7 \mathrm{G}$ & \\
\hline & $\mathrm{Zn}_{0,95} \mathrm{Co}_{0,05} \mathrm{O}$ & $4 \mathrm{M}$ & $5 \mathrm{P}$ & & $4 \mathrm{MD}$ & $8 \mathrm{P}$ & \\
\hline & $\mathrm{Zn}_{0,90} \mathrm{Co}_{0,10} \mathrm{O}$ & $4 \mathrm{M}$ & $7 \mathrm{P}$ & & $4 \mathrm{MD}$ & $8 \mathrm{G}$ & \\
\hline & $\mathrm{Zn}_{0,85} \mathrm{Co}_{0,15} \mathrm{O}$ & $4 \mathrm{M}$ & $8 \mathrm{G}$ & & $4 \mathrm{MD}$ & $7 \mathrm{G}$ & \\
\hline & $\mathrm{Zn}_{0,80} \mathrm{Co}_{0,20} \mathrm{O}$ & $4 \mathrm{M}$ & $8 \mathrm{G}$ & & $4 \mathrm{MD}$ & $8 \mathrm{P}$ & \\
\hline \multirow[t]{6}{*}{10} & $\mathrm{ZnO}$ comercial & $4 \mathrm{M}$ & $7 \mathrm{G}$ & & $8 \mathrm{MD}$ & $7 \mathrm{P}$ & \\
\hline & $\mathrm{ZnO}$ & $4 \mathrm{MD}$ & $6 \mathrm{G}$ & & $4 \mathrm{M}$ & $8 \mathrm{G}$ & \\
\hline & $\mathrm{Zn}_{0,95} \mathrm{Co}_{0,05} \mathrm{O}$ & $6 \mathrm{M}$ & $7 \mathrm{P}$ & & $8 \mathrm{MD}$ & $8 \mathrm{P}$ & \\
\hline & $\mathrm{Zn}_{0,90} \mathrm{Co}_{0,10} \mathrm{O}$ & $4 \mathrm{M}$ & $7 \mathrm{G}$ & & $8 \mathrm{MD}$ & $8 \mathrm{P}$ & \\
\hline & $\mathrm{Zn}_{0,85} \mathrm{Co}_{0,15} \mathrm{O}$ & $6 \mathrm{M}$ & $7 \mathrm{G}$ & & $8 \mathrm{MD}$ & $8 \mathrm{P}$ & \\
\hline & $\mathrm{Zn}_{0,80} \mathrm{Co}_{0,20} \mathrm{O}$ & $4 \mathrm{~F}$ & $7 \mathrm{G}$ & & $8 \mathrm{MD}$ & $9 \mathrm{P}$ & \\
\hline \multirow[t]{5}{*}{20} & $\mathrm{ZnO}$ & $4 \mathrm{~F}$ & $8 \mathrm{G}$ & & $8 \mathrm{M}$ & $8 \mathrm{P}$ & \\
\hline & $\mathrm{Zn}_{0,95} \mathrm{Co}_{0,05} \mathrm{O}$ & $4 \mathrm{~F}$ & $8 \mathrm{G}$ & & $8 \mathrm{MD}$ & $8 \mathrm{P}$ & \\
\hline & $\mathrm{Zn}_{0,90} \mathrm{Co}_{0,10} \mathrm{O}$ & $4 \mathrm{M}$ & $8 \mathrm{G}$ & & $8 \mathrm{MD}$ & $8 \mathrm{P}$ & \\
\hline & $\mathrm{Zn}_{0,85} \mathrm{Co}_{0,15} \mathrm{O}$ & $4 \mathrm{~F}$ & $7 \mathrm{G}$ & & $8 \mathrm{MD}$ & $8 \mathrm{P}$ & \\
\hline & $\mathrm{Zn}_{0,80} \mathrm{Co}_{0,20} \mathrm{O}$ & $8 \mathrm{~F}$ & $9 \mathrm{P}$ & & $8 \mathrm{M}$ & $8 \mathrm{P}$ & \\
\hline
\end{tabular}


TABLA 7. Icorr y eficiencia de inhibición de los pigmentos estudiados

\begin{tabular}{lcc}
\hline Óxido & $\begin{array}{c}\text { Icorr } \\
\left(\boldsymbol{\mu} \mathbf{A} \cdot \mathbf{c m}^{-2}\right)\end{array}$ & $\begin{array}{c}\text { Eficiencia de } \\
\text { Inhibición }(\%)\end{array}$ \\
\hline Blanco (sin pigmento) & 86,70 & -- \\
$\mathrm{ZnO}$ comercial & 31,76 & 63,36 \\
$\mathrm{ZnO}$ & 13,38 & 84,57 \\
$\mathrm{Zn}_{0,95} \mathrm{Co}_{0,05} \mathrm{O}$ & 12,70 & 85,35 \\
$\mathrm{Zn}_{0,90} \mathrm{Co}_{0,10} \mathrm{O}$ & 7,479 & 91,37 \\
$\mathrm{Zn}_{0,85} \mathrm{Co}_{0,15} \mathrm{O}$ & 6,669 & 92,31 \\
$\mathrm{Zn}_{0,80} \mathrm{Co}_{0,20} \mathrm{O}$ & 3,270 & 96,23 \\
\hline
\end{tabular}

- Análisis de los óxidos por microscopía electrónica de transmisión:

Los pigmentos sintetizados, de forma cuasi-rectangular, pueden ser considerados nanopartículas debido al tamaño que presentan, por debajo de los $100 \mathrm{~nm}$.

\section{- Ensayos de corrosión acelerada:}

Como se puede apreciar, los sistemas alquídicos presentaron mejor desempeño con el aumento del contenido de los pigmentos (óxidos mixtos), con relación al peso de la pintura, para el ensayo de niebla salina neutra. Desde 5\%, donde la corrosión es bastante visible, hasta $20 \%$, donde la corrosión es menos apreciable. El aumento de la capacidad inhibidora del óxido mixto con el incremento del contenido de cobalto es evidente en los imprimantes alquídicos para cada porcentaje en peso de pigmento por peso de pintura. Por otro lado, algunas probetas presentan daños provenientes de sus bordes, lo cual influyó en los grados de herrumbre y ampollamiento. Esto fue observado en las probetas de $10 \%$ en pigmento y contenidos de cobalto mayores.

Para el caso del ensayo en dióxido de azufre, el incremento del contenido de los pigmentos (óxidos mixtos) mejora el desempeño de los imprimantes alquídicos. En este caso, el aumento de la capacidad inhibidora del óxido mixto con el incremento del contenido de cobalto no fue tan marcado como en el ensayo en niebla salina neutra (5 y $20 \%$ de óxido mixto en la pintura); sin embargo, para $10 \%$ en peso de pigmento (óxido mixto) fue apreciada una tendencia similar a la observada en el ensayo de niebla salina.

\section{- Ensayos electroquímicos:}

Se puede apreciar una diferencia mayor al $50 \%$ entre los valores de Icorr del Blanco y del $\mathrm{ZnO}$ comercial, lo cual indica que el $\mathrm{ZnO}$ tiene propiedades inhibidoras de la corrosión del acero en el medio salino evaluado. Asimismo, se puede apreciar una diferencia mayor al 50\% entre los valores de Icorr del $\mathrm{ZnO}$ comercial y el nano, lo cual demuestra que la capacidad inhibidora del $\mathrm{ZnO}$ es mayor para las partículas en tamaño nano. Por último, a medida que aumenta el contenido de cobalto en el óxido mixto los valores de Icorr tienden a disminuir.

De manera similar, la eficiencia de inhibición aumenta a partir del $\mathrm{ZnO}$ comercial $(63,36 \%)$ hasta el pigmento con mayor contenido de cobalto $(96,23 \%)$. Además, la eficiencia de inhibición aumenta en mayor proporción al pasar del $\mathrm{ZnO}$ comercial al $\mathrm{ZnO}$ nano.

\section{CONCLUSIONES}

Los pigmentos sintetizados de óxidos de zinc y cobalto en tamaño nanométrico fueron evaluados en su capacidad anticorrosiva.

- La estequiometría de los óxidos $\mathrm{Zn}_{0,95} \mathrm{Co}_{0,05} \mathrm{O}$ - $\mathrm{Zn}_{0,90} \mathrm{Co}_{0,10} \mathrm{O}-\mathrm{Zn}_{0,85} \mathrm{Co}_{0,15} \mathrm{O}-\mathrm{Zn}_{0,80} \mathrm{Co}_{0,20} \mathrm{O}$ obtenidos mediante síntesis por combustión en solución fue confirmada mediante análisis químico elemental del $\mathrm{Zn}$ y Co utilizando el equipo ICP-OES. Las fases $\mathrm{ZnO}$ y $\mathrm{Co}_{3} \mathrm{O}_{4}$ en los óxidos mixtos fueron identificadas mediante Difracción de Rayos X, mostrando cualitativamente en los espectros el incremento de la fase $\mathrm{Co}_{3} \mathrm{O}_{4}$ conforme aumenta el contenido de cobalto de 5 a $20 \%$ en el óxido mixto $\mathrm{Zn}_{1-\mathrm{x}} \mathrm{Co}_{\mathrm{x}} \mathrm{O}$. Posteriormente, en los ensayos TEM pudo ser observado el tamaño nanométrico y la forma cuasi-rectangular de los pigmentos sintetizados.

- Los ensayos de corrosión acelerada (niebla salina y dióxido de Azufre) realizados sobre probetas pintadas con imprimantes alquídicos que contienen los pigmentos $\mathrm{Zn}_{1-\mathrm{x}} \mathrm{Co}_{\mathrm{x}} \mathrm{O}$ sintetizados confirman el incremento de protección ofrecida por el óxido mixto con el aumento del contenido de cobalto. Las tendencias observadas en los ensayos de corrosión acelerada han sido confirmadas por los ensayos electroquímicos, según los cuáles la capacidad inhibidora de la corrosión del acero aumenta cuando el pigmento es usado en tamaño nanométrico y cuando es incrementado el contenido de cobalto en los mismos.

\section{AGRADECIMIENTOS}

Los autores del presente trabajo de investigación deseamos agradecer a la Dirección de Gestión de Investigación de la Pontificia Universidad Católica del Perú (PUCP) por haber financiado parte del estudio. Asimismo, deseamos expresar nuestro agradecimiento al Instituto de Corrosión y Protección de la PUCP por proveer de los recursos necesarios en infraestructura, equipamiento y personal de apoyo para la ejecución del trabajo experimental. Por último, deseamos agradecer a la Dra. 
Maribel Guzmán (PUCP) y a la Universidad de Lovaina por los análisis de Microscopía Electrónica de Transmisión.

\section{REFERENCIAS}

Abu Ayana, Y.M., El-Sawy, S.M., Salah, S.H. (1997). Zincferrite pigment for corrosion protection. Anti-Corrosion Methods and Materials 44 (6), 381-388. https://doi. org/10.1108/00035599710367681.

Ahmed, N.M., Selim, M.M. (2005). The effect of cobalt oxide on zinc oxide in a new anticorrosive green pigment. AntiCorrosion Methods and Materials 52 (6), 353-364. https:// doi.org/10.1108/00035590510624695.

Ammar. Sh., Ramesh, K., Vengadaesvaran, B., Ramesh, A.K., Arof, A.K. (2016a). Amelioration of anticorrosion and hydrophobic properties of epoxy/PDMS composite coatings containing nano $\mathrm{ZnO}$ particles. Prog. Org. Coat. 92, 54-65. https://doi.org/10.1016/j.porgcoat.2015.12.007.

Ammar, Sh., Ramesh, K, Vengadaesvaran, B., Ramesh, S., Arof, A.K. (2016b). Formulation and characterization of hybrid polymeric/ $\mathrm{ZnO}$ nanocomposite coatings with remarkable anti-corrosion and hydrophobic characteristics. J. Coat. Technol. Res. 13 (5), 921-930. https://doi. org/10.1007/s11998-016-9799-z.

Aruna, S.T., Mukasyan, A.S. (2008). Combustion synthesis and nanomaterials. Curr. Opin. Solid St. Mat. Sci. 12 (3-4), 44-50. https://doi.org/10.1016/j.cossms.2008.12.002.

ASTM D 714-02 (2009). Standard Test Method for Evaluating Degree of Blistering of Paints. ASTM International, West Conshohocken, PA, USA.

ASTM D 610-08 (2012). Standard Practice for Evaluating Degree of Rusting on Painted Steel Surfaces. ASTM International, West Conshohocken, PA, USA

ASTM B 499-09 (2014). Standard Test Method for Measurement of Coating Thicknesses by the Magnetic Method: Nonmagnetic Coatings on Magnetic Basis Metals. ASTM International, West Conshohocken, PA, USA

ASTM D 562-10 (2014). Standard Test Method for Consistency of Paints Measuring Krebs Unit (KU) Viscosity Using a Stormer-Type Viscometer. ASTM International, West Conshohocken, PA, USA.

ASTM B 117-16 (2016). Standard Practice for Operating Salt Spray (Fog) Apparatus. ASTM International, West Conshohocken, PA, USA

Cayton, R.H., Sawitowski, T. (2005). The Impact of NanoMaterials on Coatings Technologies. Technical Proceedings NSTI-Nanotech 2005. Vol. 2, Chapter 2, Nano Composites. https://www.nsti.org/publications/Nanotech/ 2005/pdf/829.pdf.
Dhoke, S.K., Khanna, A.S., Mangal Sinha, T.J. (2009). Effect of nano-ZnO particles on the corrosion behavior of alkyd-based waterborne coatings. Prog. Org. Coat. 64 (4), 371-382. https://doi.org/10.1016/j.porgcoat.2008. 07.023 .

Dhoke, S.K., Khanna, A.S. (2009a). Effect of nano- $\mathrm{Fe}_{2} \mathrm{O}_{3}$ particles on the corrosion behavior of alkyd-based waterborne coatings. Corros. Sci. 51 (1), 6-20. https://doi.org/10.1016/j. corsci.2008.09.028.

Dhoke, S.K., Khanna, A.S. (2009b). Electrochemical behavior of nano-iron oxide modified alkyd based waterborne coatings. Mater. Chem. Phys. 117 (2-3), 550-556. https://doi. org/10.1016/j.matchemphys.2009.07.010.

Koleske, J.V. (1995). Paint and Coating Testing Manual. ASTM, 14th Edition, Philadelphia.

Patil, K.C. (1993). Advanced ceramics: Combustion synthesis and properties. Bull. Mater. Sci. 16 (6), 533-541. https:// doi.org/10.1007/BF02757654.

Patil, K.C., Aruna, S.T., Ekambaram, S. (1997). Combustion synthesis. Curr. Opin. Solid St. Mat. Sci. 2 (2), 158-165. https://ac.els-cdn.com/S1359028697800605/1-s2.0S1359028697800605-main.pdf? tid=82757472-ff56-449b9b44-27e5ee7a5ca9\&acdnat $=1550661066 \_5 \mathrm{~b} 882 \mathrm{c} 3 \mathrm{caa} 4391$ 2be2f21454b4a9bf29.

Patil, K.C., Hedge, M.S., Tanu Rattan, Aruna, S.T. (2008). Chemistry of Nanocrystalline Oxide Materials. Combustion Synthesis, Properties and Applications. World Scientific, New Jersey.

Ramezanzadeh, B., Attar, M.M., Farzam, M. (2011). A study on the anticorrosion performance of the epoxy-polyamide nanocomposites containing $\mathrm{ZnO}$ nanoparticles. Prog. Org. Coat. 72 (3), 410-422. https://doi.org/10.1016/j.porgcoat. 2011.05.014.

Rashvand, M., Ranjbar, Z. (2013). Effect of nano-ZnO particles on the corrosion resistance of polyurethane-based waterborne coatings immersed in sodium chloride solution via EIS technique. Prog. Org. Coat. 76 (10), 1413-1417. https:// doi.org/10.1016/j.porgcoat.2013.04.013

Rasouli, S., Danaee, I. (2011). Effect of preparation method on the anti-corrosive properties of nanocrystalline $\mathrm{Zn}-\mathrm{CoO}$ ceramic pigments. Mater. Corros. 62 (5), 405-410. https:// doi.org/10.1002/maco.201005758.

USEPA (2000a). Cobalt Compounds. United States Environmental Protection Agency. Consulta: 20 de febrero de 2019.

USEPA (2000b). Chromium Compounds. United States Environmental Protection Agency. Consulta: 20 de febrero de 2019. https://www.epa.gov/sites/production/files/2016-09/documents/chromium-compounds.pdf.

USEPA (2018). Health Effects Notebook for Hazardous Air Pollutants. United States Environmental Protection Agency. Consulta: 20 de febrero de 2019.https://www.epa.gov/haps/ health-effects-notebook-hazardous-air-pollutants. 